\title{
Nucleotide Variations in microRNA-Binding Sites: The Need of Novel Tools for the Nucleic Acid Alignment
}

\author{
Jill Zhong ${ }^{1}$, Holly Clifton ${ }^{2}$ and Alexander Kofman*2 \\ ${ }^{1}$ Department of Computer Sciences, Troy University, AL, USA \\ ${ }^{2}$ Department of Biological \& Environmental Sciences, Troy University, AL, USA
}

*Corresponding author: Alexander Kofman, Department of Biological \& Environmental Sciences, 210-D MSCX (McCall Hall), Troy University, AL 36082, U.S.A

\section{ARTICLE INFO}

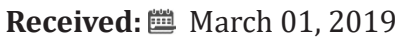

Published: March 11, 2019

Citation: Jill Zhong, Holly Clifton, Alexander Kofman. Nucleotide Variations in microRNA-Binding Sites: The Need of Novel Tools for the Nucleic Acid Alignment. Biomed J Sci \& Tech Res 15(4)-2019. BJSTR. MS.ID.002747.

\begin{abstract}
It has been proposed that naturally-occurring variations in microRNAs and their target sites on messenger RNAs may be linked with various human pathologies, including cancer. The growing number of reports clearly indicates the need of the development of complementary sequence alignment algorithms, where the interaction of two complementary RNAs (specifically microRNA and its target mRNA) can be assessed in the context of the multiple single nucleotide polymorphisms.
\end{abstract}

Keywords: MicroRNA; Nucleotide; Variations; Polymorphisms; Alignment; Algorithm

Abbreviations: mRNA: Messenger RNA; SNP: Single Nucleotide Polymorphism

\section{Introduction}

During the last fifteen years, the attention of biologists and medical scientists has been attracted to microRNAs, the small regulatory molecules of about 22 nucleotides long. The suppressive effect of microRNAs on gene expression is well-known and generally attributed to microRNA-mediated degradation of its target messenger RNA (mRNA, the molecule produced by the gene), messenger RNA translational repression, or both. To accomplish this, microRNA aligns with its mRNA target upon the principles of complementarity between the microRNA and mRNA nucleotides [1] (Figure 1). It has been suggested that naturally-occurring variations in microRNAs and their target genes may be associated with various human pathologies including cancer [2,3]. Even substitutions of only one nucleotide, the so-called Single Nucleotide Polymorphisms (SNPs) can either destroy or create microRNAbinding sites, thus, altering microRNA ability to target oncogenes and rendering tumor-suppressor genes susceptible to microRNAmediated inhibition. By now, all human microRNA-binding sites for all microRNAs and their target mRNAs have been predicted with the help of the various software applications and compiled into the databases.

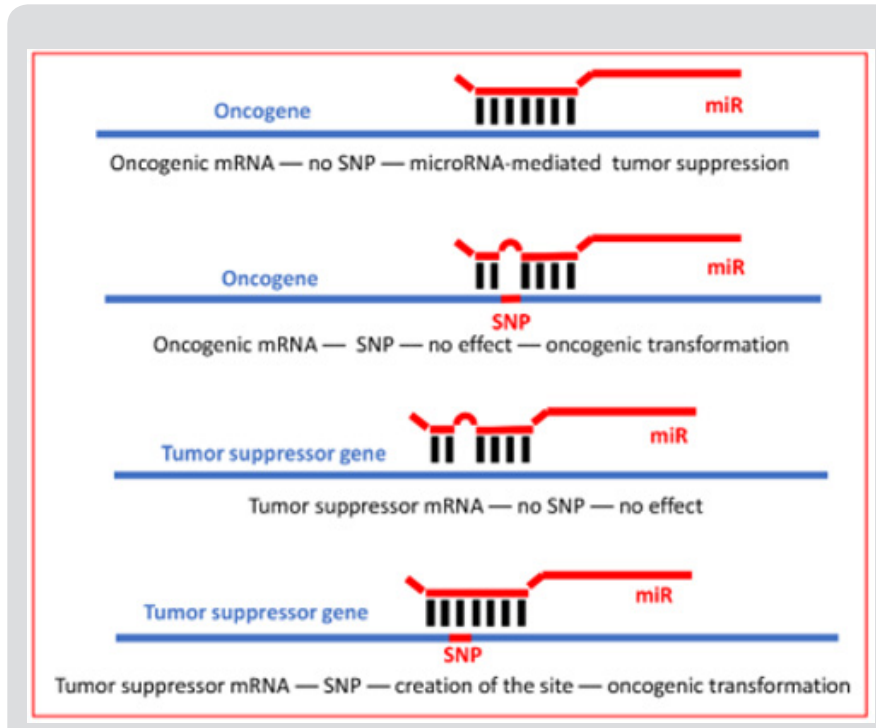

Figure 1: Destruction and creation of microRNA-binding sites by SNP; implications for oncogenesis 
However, the computer-assisted prediction of microRNAbinding sites relied exclusively on one or few reference genomic sequences and did not take into consideration the significant variations within human genomes. In meantime, SNPs are reported to occur at a rate of about 1 per $300-1000$ bases in the individual human genome [4] and with the frequency of $1 \%$ and higher in a population [5,6]. The number of SNP in the human genome was estimated to be around $1.5 \times 10^{6}$ per individual [7]. However, the advances of NextGen Sequencing technologies revealed that some previously believed to be rare mutations exceeded the frequency threshold set at $1 \% 6$. Previously, we demonstrated that even the only "seed" regions of microRNA-binding sites can harbor as many as 4 SNPs, and their coincidence may result in hyper-functional or completely disabled microRNA-binding sites with the following significant phenotypic variations and predisposition to cancers [8]

\section{Conclusion}

The existing data do not take into consideration the significant variability of microRNA-binding sites due to abundancy of SNPs. In addition, there is no possibility to predict the creation of the new microRNA-binding sites upon the variations of single nucleotides in the regions, where microRNA-binding sites are absent. Finally, there are no software tools to quickly assess the probability of hyperfunctional or completely disabled microRNA-binding sites based on the known frequencies of SNPs. Altogether, these problems warrant the development of algorithms that allow biomedical researchers to align the individual microRNA and mRNA sequences based on their Watson-Crick complementarity in order to predict how SNPs may weaken or enhance the known microRNA-binding sites, or to create microRNA-binding sites de novo.

\section{References}

1. Kofman AV, Letson C, Dupart E, Bao Y, Newcomb WW, et al. (2013) The p53-microRNA-34a axis regulates cellular entry receptors for tumorassociated human herpes viruses. Med Hypotheses 81: 62-67.

2. Chen K, Song F, Calin GA, Wei Q, Hao X, et al. (2008) Polymorphisms in microRNA targets: A gold mine for molecular epidemiology. Carcinogenesis 29: 1306-1311.

3. Sethupathy P, Collins FS (2008) MicroRNA target site polymorphisms and human disease. Trends Genet 24: 489-497.

4. Sethupathy P, Borel C, Gagnebin M, Grant GR, Deutsch S, et al. (2007) Human microRNA-155 on chromosome 21 differentially interacts with its polymorphic target in the AGTR1 3' untranslated region: A mechanism for functional single-nucleotide polymorphisms related to phenotypes. Am J Hum Genet 81: 405-413.

5. Karki R, Pandya D, Elston RC, Ferlini C (2015) Defining "mutation" and "polymorphism" in the era of personal genomics. BMC Med Genomics 8: 37.

6. Manikandan M, Munirajan AK (2014) Single nucleotide polymorphisms in microRNA binding sites of oncogenes: Implications in cancer and pharmacogenomics. OMICS 18: 142-154.

7. Sachidanandam R, Weissman D, Schmidt SC, Kakol JM, Stein LD, et al. (2001) A map of human genome sequence variation containing 1.42 million single nucleotide polymorphisms. Nature 409: 928-933.

8. Amber Budmark MC, Tyrel Haley, Brady Hicks, Maria Koenen, Thea Patrick, et al. (2018) Single nucleotide variations within and around microRNA-binding sites. Cancer Research 78: 490.
ISSN: 2574-1241

DOI: 10.26717/BJSTR.2019.15.002747

Alexander Kofman. Biomed J Sci \& Tech Res

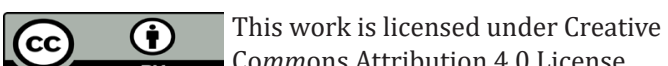

Submission Link: https://biomedres.us/submit-manuscript.php

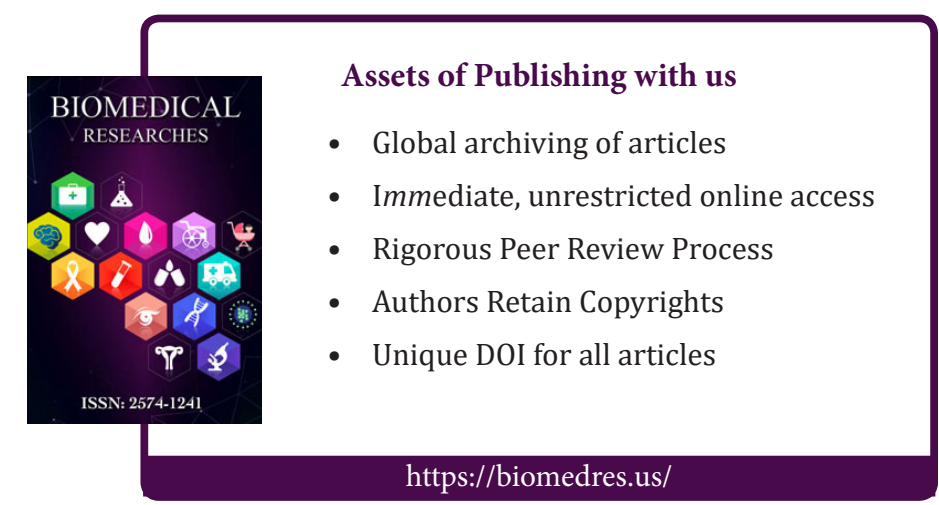

